\title{
The generalized Korteweg-de Vries equation with time oscillating nonlinearity in scale critical Sobolev space
}

\author{
Jun-ichi Segata and Keishu Watanabe
}

\begin{abstract}
We consider the generalized Korteweg-de Vries (gKdV) equation with the time oscillating nonlinearity:

$$
\partial_{t} u+\partial_{x}^{3} u+g(\omega t) \partial_{x}\left(|u|^{p-1} u\right)=0, \quad(t, x) \in \mathbb{R} \times \mathbb{R} .
$$

Under the suitable assumption on $g$, we show that if the nonlinear term is mass critical or supercritical i.e., $p \geq 5$ and $u(0) \in \dot{H}^{s_{p}}$, where $s_{p}=$ $1 / 2-2 /(p-1)$ is a scale critical exponent, then there exists a unique global solution to $(\mathrm{gKdV})$ provided that $|\omega|$ is sufficiently large. We also obtain the behavior of the solution to $(\mathrm{gKdV})$ as $|\omega| \rightarrow \infty$.
\end{abstract}

Mathematics Subject Classification. Primary 35Q53; Secondary 35Q35, 35B40.

Keyword. Generalized Korteweg-de Vries equation.

\section{Introduction}

We consider the generalized Korteweg-de Vries ( $\mathrm{gKdV}$ ) equation with the time oscillating nonlinearity:

$$
\begin{cases}\partial_{t} u+\partial_{x}^{3} u+g(\omega t) \partial_{x}\left(|u|^{p-1} u\right)=0, & (t, x) \in \mathbb{R} \times \mathbb{R} \\ u(0, x)=\phi(x), & x \in \mathbb{R}\end{cases}
$$

where $u: \mathbb{R} \times \mathbb{R} \rightarrow \mathbb{R}$ is an unknown function, $\phi: \mathbb{R} \rightarrow \mathbb{R}$ is a given function and $p>1$. The function $g \in C^{1}(\mathbb{R} ; \mathbb{R})$ is a given periodic function with period $L>0$.

The class of equations (1.1) arises in several fields of physics. Equation (1.1) is a generalization of the notable Korteweg-de Vries equation which models long waves propagating in a channel [16] and the transitional KdV equation which describes the solitary waves propagating on the thermocline in a lake [14].

J. Segata is partially supported by MEXT, Grant-in-Aid for Young Scientists (A) 25707004. 
For the case where $g$ is constant, the local well-posedness of the initial value problem (1.1) in a scale subcritical Sobolev space $H^{s}(\mathbb{R}), s>s_{p}$ has been studied by many authors $[2,9-13,20,24]$, where $s_{p}=1 / 2-2 /(p-1)$ is a scale critical exponent. A fundamental work on the local well-posedness is due to Kenig-Ponce-Vega [12]. They proved that (1.1) is locally well-posed in $H^{s}(\mathbb{R})$ with $s>3 / 4(p=2), s \geq 1 / 4(p=3), s \geq 1 / 12(p=4)$ and $s \geq s_{p}(p \geq 5)$. Concerning the well-posedness of (1.1) in the scale critical $\dot{H}^{s_{p}}$ space, Kenig-Ponce-Vega [12] proved the local and (small data) global wellposedness when $p \geq 5$. Notice that Farah-Pastor [9] simplified Kenig-PonceVega's proof. Later on, the above results were extended to a homogeneous Besov space $\dot{B}_{2, \infty}^{s_{p}}$ by Strunk $[24](p \geq 5)$.

Since the proof in [12] also works for the case where $g$ is not constant, the local well-posedness for (1.1) with a non constant $g$ follows from [12]. On the other hand, Nunes [22] showed the global existence for (1.1) with $p=2$ in $H^{1}(\mathbb{R})$ under the assumption that $g \in C(\mathbb{R} ; \mathbb{R})$ and $g^{\prime} \in L_{\text {loc }}^{1}(\mathbb{R})$. Notice that Nunes's proof is based on the almost conservation quantities for (1.1) and applicable for the arbitrary large data in $H^{1}$ for the defocusing case, i.e, $g$ is strictly negative and for the focusing case, i.e, $g$ is strictly positive with the mass sub-critical exponent $1<p<5$. Furthermore, in Martel-Merle [18], a family of solutions to (1.1) which blow up in finite time was constructed for the case where $g$ is positive constant (focusing) and $p=5$ (mass critical).

In this paper, we study the global existence and the behavior of solution $u_{\omega}$ to $(1.1)$ as $|\omega| \rightarrow \infty$. We first review the known results on the nonlinear Schrödinger equation with the time oscillating nonlinearity:

$$
\begin{cases}i \partial_{t} u+\Delta u+g(\omega t)\left(|u|^{p-1} u\right)=0, & (t, x) \in \mathbb{R} \times \mathbb{R}^{n}, \\ u(0, x)=\phi(x), & x \in \mathbb{R}^{n},\end{cases}
$$

where $p>1, u: \mathbb{R}^{n} \times \mathbb{R} \rightarrow \mathbb{C}$ is an unknown function, $\phi: \mathbb{R}^{n} \rightarrow \mathbb{C}$ is a given function, and $g \in C^{1}(\mathbb{R} ; \mathbb{R})$ is a given periodic function with a period $L>0$. Abdullaev-Caputo-Kraeukel-Malomed [1] and Konotop-Pacciani [15] investigated the effect of the time oscillation term $g(\omega t)$ in the global behavior of solution to (1.2) via the numerical methods. Cazenave-Scialom [4] proved that if $\phi \in H^{1}\left(\mathbb{R}^{n}\right)$ and $1+4 / n \leq p<p^{*}$ with $p^{*}=\infty$ if $n=1,2$ and $p^{*}=1+4 /(n-2)$ if $n \geq 3$, then, the solution $u_{\omega}$ converges to the solution $U$ to

$$
\begin{cases}i \partial_{t} U+\Delta U+m(g)|U|^{p-1} U=0, & (t, x) \in \mathbb{R} \times \mathbb{R}^{n}, \\ U(0, x)=\phi(x), & x \in \mathbb{R}^{n}\end{cases}
$$

as $|\omega| \rightarrow \infty$, where $m(g)$ is given by

$$
m(g)=\frac{1}{L} \int_{0}^{L} g(s) d s .
$$

Furthermore, Fang-Han [8] showed the similar result for the case where $\phi \in$ $H^{1}\left(\mathbb{R}^{n}\right)$ and $p$ is energy critical, i.e., $p=1+4 /(n-2)(n \geq 3)$. DamergiGoubet [6] showed that the oscillations do not prevent the blow up for any $\omega \in \mathbb{R}$ for the case where $n=2, p \geq 3$ and $g(\omega t)=\cos ^{2}(\omega t)$. 
Concerning the generalized $\mathrm{KdV}$ equation (1.1), Carvajal-PantheeScialom [3] have shown that if $\phi \in H^{1}(\mathbb{R})$ and $p$ is mass critical, i.e., $p=5$, then the solution $u_{\omega}$ to $(1.1)$ converges to the solution $U$ to

$$
\begin{cases}\partial_{t} U+\partial_{x}^{3} U+m(g) \partial_{x}\left(|U|^{p-1} U\right)=0, & (t, x) \in \mathbb{R} \times \mathbb{R}, \\ U(0, x)=\phi(x), & x \in \mathbb{R}\end{cases}
$$

as $|\omega| \rightarrow \infty$, where $m(g)$ is given by (1.4). Moreover, Panthee-Scialom [23] proved the similar result for the case where $\phi \in H^{1}(\mathbb{R})$ and $p$ is mass supercritical, i.e., $p>5$.

In the present paper, we shall show that if $\phi \in \dot{H}^{s_{p}}(\mathbb{R}), s_{p}=1 / 2-2$ / $(p-1)$, and $p$ is mass critical or supercritical, i.e., $p \geq 5$, then the solution $u_{\omega}$ to (1.1) exists globally and converges to the solution $U$ to (1.5) as $|\omega| \rightarrow \infty$. Since $H^{1} \subset \dot{H}^{s_{p}}$, our result is an improvement of $[3,23]$ for the case $p \geq 5$.

Before we state our main theorem, we introduce several notations. Throughout this paper, we denote $s_{p}:=1 / 2-2 /(p-1)$. For $T \in(0, \infty]$, the norm $\|\cdot\|_{X_{T}}$ is defined by

$$
\|f\|_{X_{T}}:=\|f\|_{L_{T}^{\infty} \dot{H}^{s_{p}}}+\left\|\left|\partial_{x}\right|^{s_{p}} f\right\|_{L_{x}^{5} L_{T}^{10}}+\|f\|_{L_{x}^{\frac{5}{4}(p-1)} L_{T}^{\frac{5}{2}(p-1)}},
$$

where $\|f\|_{L_{T}^{p}}=\|f\|_{L_{(0, T)}^{p}}$. The norm defined by (1.6) is used in Farah-Pastor [9]. Let $\{S(t)\}_{t \in \mathbb{R}}$ be a unitary group generated by $-\partial_{x}^{3}$ :

$$
(S(t) \phi)(x)=\frac{1}{\sqrt{2 \pi}} \int_{-\infty}^{\infty} e^{i x \xi+i t \xi^{3}} \hat{\phi}(\xi) d \xi .
$$

Definition 1.1. (Solution) Let $T \in(0, \infty]$. We say that $u$ is a (mild) solution to (1.1) on $[0, T)$ in $\dot{H}^{s_{p}}$ if $u \in C\left([0, T) ; \dot{H}^{s_{p}}(\mathbb{R})\right) \cap X_{T}$ and satisfies

$$
u(t)=S(t) \phi-\int_{0}^{t} S\left(t-t^{\prime}\right) g\left(\omega t^{\prime}\right) \partial_{x}\left(|u|^{p-1} u\right)\left(t^{\prime}\right) d t^{\prime} .
$$

The solution to (1.5) is defined in a similar way.

For the solution $u$ to (1.1), we define

$$
T_{\max }:=\sup \{T \in(0, \infty] \mid \text { Solution } u \text { to }(1.1) \text { can be extended to }[0, T)\} \text {. }
$$

We say that $u$ is a maximal solution to (1.1) if $u$ is a solution to (1.1) on $\left[0, T_{\max }\right)$. Similarly, for the solution $U$ to $(1.5)$, we define

$$
S_{\text {max }}:=\sup \{T \in(0, \infty] \mid \text { Solution } U \text { to }(1.5) \text { can be extended to }[0, T)\} \text {. }
$$

We say that $U$ is a maximal solution to (1.5) if $U$ is a solution to (1.5) on $\left[0, S_{\max }\right)$.

The main result of this paper is as follows.

Theorem 1.2. Assume $p \geq 5$ and $\phi \in \dot{H}^{s_{p}}(\mathbb{R})$. Let $u_{\omega}$ be a maximal solution to (1.1) and let $U$ be a solution to (1.5) on maximal interval $\left[0, S_{\max }\right)$. Furthermore, we assume $S_{\max }=\infty$ and

$$
\|U\|_{L_{x}^{\frac{5}{4}(p-1)} L_{t}^{\frac{5}{2}(p-1)}}<\infty .
$$


Then there exists $\omega_{0}>0$ such that if $\omega$ satisfies $|\omega|>\omega_{0}$, then $u_{\omega}$ is global solution to (1.1). Moreover, we have

$$
\left\|u_{\omega}-U\right\|_{X_{\infty}} \longrightarrow 0 \quad \text { as }|\omega| \rightarrow \infty .
$$

The proof of Theorem 1.2 is essentially based on the arguments due to Cazenave-Scialom [4] for the nonlinear Schrödinger equation (1.2) and Carvajal-Panthee-Scialom [3] and Panthee-Scialom [23] for the generalized $\mathrm{KdV}$ equation (1.1). Their proofs are based on the combination of the Strichartz estimate and the Gronwall type lemma. It is not likely that the Gronwall type lemma used in [4] works for the scaling critical space. Instead of the Gronwall type lemma, we divide the time interval into subintervals to obtain various estimates need to prove Theorem 1.2. Although this kind of technique is used for the energy critical nonlinear Schrödinger equation (see [8] for example), we meet some technical difficulties due to the presence of $L_{x}^{p} L_{T}^{q}$ type norms. We overcome this difficulty by modifying the argument used in Killip-Kwon-Shao-Visan [17, Theorem 3.1].

We give two examples of $g$ and $\phi$ satisfying the assumptions of Theorem 1.2.

Example. Let $p \geq 5$ and $m(g)=0$. In this case, Eq. (1.5) is "linear" (i.e., Airy equation). By the space-time estimates for the solution to the Airy equation (see Proposition 2.5 below), we see that for any $\phi \in \dot{H}^{s_{p}}(\mathbb{R})$, the solution $U$ to (1.5) exists globally in time and satisfies the assumption (1.7) in Theorem 1.2.

Example. Let $p=5$ and $m(g)<0$. In this case, Dodson [7] showed that for any $\phi \in L^{2}(\mathbb{R})\left(=H^{s_{5}}(\mathbb{R})\right)$ there uniquely exists a global solution $U$ to $(1.5)$ satisfying the assumption (1.7) in Theorem 1.2.

Combing Theorem 1.2 and the above examples, we obtain the following:

Corollary 1.3. Let $p \geq 5$. Suppose that $g$ satisfies $m(g)=0$. Then there exists $\omega_{0}>0$ such that if $\omega$ satisfies $|\omega|>\omega_{0}$, then for any $\phi \in \dot{H}^{s_{p}}(\mathbb{R})$, there exists a unique global solution $u_{\omega} \in C\left([0, \infty) ; \dot{H}^{s_{p}}(\mathbb{R})\right) \cap X_{\infty}$ to $(1.1)$.

Corollary 1.4. Let $p=5$. Suppose that $g$ satisfies $m(g)<0$. Then there exists $\omega_{0}>0$ such that if $\omega$ satisfies $|\omega|>\omega_{0}$, then for any $\phi \in L^{2}(\mathbb{R})$, there exists a unique global solution $u_{\omega} \in C\left([0, \infty) ; L^{2}(\mathbb{R})\right) \cap X_{\infty}$ to $(1.1)$.

The plan of this paper is as follows. In Sect. 2, we prove the linear estimates for the Airy equation and the nonlinear estimates for the generalized $\mathrm{KdV}$ equation, which are needed to prove Theorem 1.2. Section 3 is devoted to proving the global existence for (1.1). In Sect. 4, we prove Theorem 1.2. Finally, in Sect. 5, we consider the subdivision of the time interval used in the proof of Theorem 1.2.

Throughout this paper we use the following notations and function spaces. For $1 \leq p \leq \infty$, we denote the Hölder conjugate exponent of $p$ by $p^{\prime}$. Let $\left|\partial_{x}\right|^{s}=\left(-\partial_{x}^{2}\right)^{s / 2}$ be a Riesz potential of order $-s:\left|\partial_{x}\right|^{s}=\mathcal{F}^{-1}|\xi|^{s} \mathcal{F}$. For $1 \leq p, q \leq \infty$ and $T \in(0, \infty]$, we define the space-time norms: 


$$
\begin{aligned}
\|f\|_{L_{T}^{q} L_{x}^{p}} & =\|\| f(t, \cdot)\left\|_{L_{x}^{p}(\mathbb{R})}\right\|_{L_{t}^{q}(0, T)}, \\
\|f\|_{L_{x}^{p} L_{T}^{q}} & =\|\| f(\cdot, x)\left\|_{L_{t}^{q}(0, T)}\right\|_{L_{x}^{p}(\mathbb{R})} .
\end{aligned}
$$

We denote $\|f\|_{L_{\infty}^{q} L_{x}^{p}}$ and $\|f\|_{L_{x}^{p} L_{\infty}^{q}}$ by $\|f\|_{L_{t}^{q} L_{x}^{p}}$ and $\|f\|_{L_{x}^{p} L_{t}^{q}}$, respectively.

\section{Preliminaries}

In this section, we prove the space-time estimates for a solution to the Airy equation and the nonlinear estimates for the generalized KdV equation.

\subsection{Linear estimates}

We first give the definition of admissible triple for the Airy equation.

Definition 2.1. (Admissible triple) Let $1 \leq p, q \leq \infty$ and $\alpha \in \mathbb{R}$. We say that $(p, q, \alpha)$ is an admissible triple if $(p, q, \alpha)$ satisfies

$$
\frac{2}{p}+\frac{1}{q}=\frac{1}{2} \quad \text { and } \quad \alpha=-\frac{1}{p}+\frac{2}{q} .
$$

Lemma 2.2. Let $\left(p_{1}, q_{1}, \alpha_{1}\right)$ and $\left(p_{2}, q_{2}, \alpha_{2}\right)$ be admissible triples. Then for any $T \in(0, \infty]$, there exist positive constants $C_{1}$ and $C_{2}$ such that the inequalities

$$
\|S(t) \phi\|_{L_{T}^{\infty} L_{x}^{2}}+\left\|\left|\partial_{x}\right|^{\alpha_{1}} S(t) \phi\right\|_{L_{x}^{p_{1}} L_{T}^{q_{1}}} \leq C_{1}\|\phi\|_{L_{x}^{2}}
$$

and

$$
\begin{aligned}
& \left\|\int_{0}^{t} S\left(t-t^{\prime}\right) f(\cdot, t) d t^{\prime}\right\|_{L_{T}^{\infty} L_{x}^{2}}+\left\|\left|\partial_{x}\right|^{\alpha_{1}} \int_{0}^{t} S\left(t-t^{\prime}\right) f(\cdot, t) d t^{\prime}\right\|_{L_{x}^{p_{1}} L_{T}^{q_{1}}} \\
& \quad \leq C_{2}\left\|\left|\partial_{x}\right|^{-\alpha_{2}} f\right\|_{L_{x}^{p_{2}^{\prime}} L_{T}^{q_{2}^{\prime}}}
\end{aligned}
$$

hold for any $\phi \in L^{2}$ and $f$ satisfying $\left|\partial_{x}\right|^{-\alpha_{2}} f \in L_{x}^{p_{2}^{\prime}} L_{T}^{q_{2}^{\prime}}$, where $C_{1}$ depends only on $p_{1}, q_{1}$ and $T$, and $C_{2}$ depends only on $p_{1}, p_{2}, q_{1}, q_{2}$ and $T$.

Proof. The homogeneous estimate (2.2) follows from the combination of the Stein analytic interpolation, the Kato's smoothing effect [12, Theorem 3.5 (i)] and the Kenig-Ruiz estimate [12, Theorem 3.7 (i)]. The inhomogeneous estimates (2.3) is obtained by combining the homogeneous estimates (2.2) and the Christ-Kiselev's lemma [21, Lemma 2]. Since the proof is now standard, we omit the detail.

Lemma 2.3. (Embedding) Let $p \geq 5$ and let $\alpha_{p}, \beta_{p}, \tilde{p}, \tilde{q}$ be given by

$$
\begin{gathered}
\alpha_{p}=\frac{1}{10}-\frac{2}{5(p-1)}, \quad \beta_{p}=\frac{3}{10}-\frac{6}{5(p-1)}, \\
\frac{1}{\tilde{p}}=\frac{2}{5(p-1)}+\frac{1}{10}, \quad \frac{1}{\tilde{q}}=\frac{3}{10}-\frac{4}{5(p-1)} .
\end{gathered}
$$

Then for any $T \in(0, \infty]$, there exists a positive constant $C$ such that the inequality

$$
\|u\|_{L_{x}^{\frac{5}{4}(p-1)} L_{T}^{\frac{5}{2}(p-1)}} \leq C\left\|\left|\partial_{x}\right|^{\alpha_{p}}\left|\partial_{t}\right|^{\beta_{p}} u\right\|_{L_{x}^{\tilde{p}} L_{T}^{\tilde{q}}}
$$


holds for any $u$ satisfying $\left|\partial_{x}\right|^{\alpha_{p}}\left|\partial_{t}\right|^{\beta_{p}} u \in L_{x}^{\tilde{p}} L_{T}^{\tilde{q}}$, where the constant $C$ depends only on $p$ and $T$.

Proof. See [12, Lemma 3.15].

Lemma 2.4. Assume $p \geq 5$. Let $\alpha_{p}, \beta_{p}, \tilde{p}, \tilde{q}$ be given in Lemma 2.3 and let $\left(p_{2}, q_{2}, \alpha_{2}\right)$ be admissible triple. Then for any $T \in(0, \infty]$, there exist positive constants $C_{1}$ and $C_{2}$ such that the inequalities

$$
\left\|\left|\partial_{x}\right|^{\alpha_{p}}\left|\partial_{t}\right|^{\beta_{p}} S(t) \phi\right\|_{L_{x}^{\tilde{p}} L_{T}^{\tilde{q}}} \leq C_{1}\|\phi\|_{\dot{H}^{s_{p}}}
$$

and

$$
\left\|\left|\partial_{x}\right|^{\alpha_{p}}\left|\partial_{t}\right|^{\beta_{p}} \int_{0}^{t} S\left(t-t^{\prime}\right) f\left(t^{\prime}\right) d t^{\prime}\right\|_{L_{x}^{\tilde{p}} L_{T}^{\tilde{q}}} \leq C_{2}\left\|\left|\partial_{x}\right|^{s_{p}-\alpha_{2}} f\right\|_{L_{x}^{p_{2}^{\prime}} L_{T}^{q_{2}^{\prime}}}
$$

hold for any for any $\phi \in \dot{H}^{s_{p}}(\mathbb{R})$ and $g$ satisfying $\left|\partial_{x}\right|^{s_{p}-\alpha_{2}} f \in L_{x}^{p_{2}^{\prime}} L_{T}^{q_{2}^{\prime}}$, where $C_{1}$ depends only on $p$ and $T$, and $C_{2}$ depends only on $p, p_{2}, q_{2}$ and $T$.

Proof. See [12, Lemma 3.14].

Combining Lemmas 2.2 and 2.4, we obtain the following:

Proposition 2.5. Let $T \in(0, \infty]$ and let $X_{T}$ be given by (1.6). Then there exists a positive constants $C_{1}$ and $C_{2}$ such that the inequalities

$$
\begin{gathered}
\|S(t) \phi\|_{X_{T}} \leq C_{1}\|\phi\|_{\dot{H}^{s p}(\mathbb{R})} \\
\left\|\int_{0}^{t} S\left(t-t^{\prime}\right) \partial_{x} f\left(t^{\prime}\right) d t^{\prime}\right\|_{X_{T}} \leq C_{2}\left\|\left|\partial_{x}\right|^{s_{p}} f\right\|_{L_{x}^{1} L_{T}^{2}}
\end{gathered}
$$

hold for any $\phi \in \dot{H}^{s_{p}}(\mathbb{R})$ and $f$ satisfying $\left|\partial_{x}\right|^{s_{p}} f \in L_{x}^{1} L_{T}^{2}$, where $C_{1}$ and $C_{2}$ depend only on $p$ and $T$.

\subsection{Nonlinear estimates}

Lemma 2.6. Let $\alpha \in(0,1), \alpha_{1}, \alpha_{2} \in[0, \alpha]$ satisfy $\alpha=\alpha_{1}+\alpha_{2}$ and let $p_{1}, p_{2}$, $q_{1}, q_{2} \in(1, \infty)$ satisfy $1 / p_{1}+1 / p_{2}=1$ and $1 / q_{1}+1 / q_{2}=1 / 2$. Then for all $T \in(0, \infty]$, there exists a positive constant $C$ such that the inequality

$$
\left\|\left|\partial_{x}\right|^{\alpha}(f g)-f\left|\partial_{x}\right|^{\alpha} g-g\left|\partial_{x}\right|^{\alpha} f\right\|_{L_{x}^{1} L_{T}^{2}} \leq C\left\|\left|\partial_{x}\right|^{\alpha_{1}} f\right\|_{L_{x}^{p_{1}} L_{T}^{q_{1}}}\left\|\left|\partial_{x}\right|^{\alpha_{2}} g\right\|_{L_{x}^{p_{2}} L_{T}^{q_{2}}}
$$

holds for any $f$ and $g$ satisfying $\left|\partial_{x}\right|^{\alpha_{1}} f \in L_{x}^{p_{1}} L_{T}^{q_{1}}$ and $\left|\partial_{x}\right|^{\alpha_{2}} g \in L_{x}^{p_{2}} L_{T}^{q_{2}}$, where $C$ depends only on $\alpha_{1}, \alpha_{2}, p_{1}, p_{2}, q_{1}, q_{2}$ and $T$.

Proof. See [12, Theorem A.13].

Lemma 2.7. Assume $\alpha \in(0,1)$. Let $p, q, p_{1}, p_{2}, q_{2} \in(1, \infty)$ and $q_{1} \in(1, \infty]$ satisfy $1 / p=1 / p_{1}+1 / p_{2}$ and $1 / q=1 / q_{1}+1 / q_{2}$. We also assume $F \in C^{1}(\mathbb{R} ; \mathbb{R})$. Then for any $T \in(0, \infty]$, there exists a positive constant $C$ such that the inequality

$$
\left\|\left|\partial_{x}\right|^{\alpha} F(f)\right\|_{L_{x}^{p} L_{T}^{q}} \leq C\left\|F^{\prime}(f)\right\|_{L_{x}^{p_{1}} L_{T}^{q_{1}}}\left\|\left|\partial_{x}\right|^{\alpha} f\right\|_{L_{x}^{p_{2} L_{T}^{q_{2}}}}
$$

holds for any $f$ satisfying $F^{\prime}(f) \in L_{x}^{p_{1}} L_{T}^{q_{1}}$ and $\left|\partial_{x}\right|^{\alpha} f \in L_{x}^{p_{2}} L_{T}^{q_{2}}$, where $C$ depends only on $\alpha, p_{1}, p_{2}, q_{1}, q_{2}$ and $T$. 
Proof. See [5, Proposition 3.1] and [12, Theorem A.6]. Notice that the alternative proof of the inequality $(2.7)$ can be found in [19, Lemma 3.7].

Proposition 2.8. Let $\alpha \in[0,1)$ and $p>2$. Then for any $T \in(0, \infty]$, there exist positive constants $C_{1}$ and $C_{2}$ such that the inequalities

$$
\left\|\left|\partial_{x}\right|^{\alpha}\left(|u|^{p-1} u\right)\right\|_{L_{x}^{1} L_{T}^{2}} \leq C_{1}\|u\|_{L_{x}^{\frac{5}{4}(p-1)} L_{T}^{\frac{5}{4}(p-1)}}^{p-1}\left\|\left|\partial_{x}\right|^{\alpha} u\right\|_{L_{x}^{5} L_{T}^{10}}
$$

and

$$
\begin{aligned}
\left\|\left|\partial_{x}\right|^{\alpha}\left(|u|^{p-1} u-|v|^{p-1} v\right)\right\|_{L_{x}^{1} L_{T}^{2}} \\
\leq C_{2}\left\{\left(\|u\|_{L_{x}^{\frac{5}{4}(p-1)} L_{T}^{\frac{5}{2}(p-1)}+\|v\|_{L_{x}^{\frac{5}{4}(p-1)}}^{p-1} L_{T}^{\frac{5}{2}(p-1)}}^{p-1}\right)\left\|\left|\partial_{x}\right|^{\alpha}(u-v)\right\|_{L_{x}^{5} L_{T}^{10}}\right. \\
\quad+\left(\|u\|_{L_{x}^{\frac{5}{4}(p-1)} L_{T}^{\frac{5}{2}(p-1)}+\|v\|_{L_{x}^{\frac{5}{4}(p-1)}}^{p-2} L_{T}^{\frac{5}{2}(p-1)}}\right) \\
\left.\quad \times\left(\left\|\left|\partial_{x}\right|^{\alpha} u\right\|_{L_{x}^{5} L_{T}^{10}}+\left\|\left|\partial_{x}\right|^{\alpha} v\right\|_{L_{x}^{5} L_{T}^{10}}\right)\|u-v\|_{L_{x}^{\frac{5}{4}(p-1)} L_{T}^{\frac{5}{2}(p-1)}}\right\}
\end{aligned}
$$

hold, where the constants $C_{1}$ and $C_{2}$ depend on $\alpha, p$ and $T$.

Proof. The proof follows from an argument similar to that in [19, Lemma 3.4]. Hence we omit the detail.

\section{Proof of global existence}

In this section, we prove propositions concerning the global existence and the convergence of solution to (1.1).

We consider

$$
\begin{cases}\partial_{t} u+\partial_{x}^{3} u+h(t) \partial_{x}\left(|u|^{p-1} u\right)=0, & (t, x) \in \mathbb{R} \times \mathbb{R}, \\ u(0, x)=\phi(x), & x \in \mathbb{R},\end{cases}
$$

where $h: \mathbb{R} \rightarrow \mathbb{R}$ is a given function.

Lemma 3.1. (Local existence) Let $p \geq 5$. Then for any $\phi \in \dot{H}^{s_{p}}(\mathbb{R})$, there exist $T=T(\phi, h)>0$ and a unique solution $u \in C\left([0, T) ; \dot{H}^{s_{p}}(\mathbb{R})\right)$ to $(3.1)$ satisfying

$$
\|u\|_{X_{T}} \leq C\|\phi\|_{\dot{H}^{s p}(\mathbb{R})},
$$

where $X_{T}$ is given by (1.6). Furthermore, let $u$ be a maximal solution to (3.1) on $\left[0, T_{\max }\right)$. Then it holds one of the following:

(i) $T_{\max }=\infty$,

(ii) $T_{\max }<\infty$ and $\|u\|_{L_{x}^{\frac{5}{4}(p-1)}} L_{T_{\max }^{\frac{5}{2}(p-1)}}=\infty$.

Proof. See Kenig-Ponce-Vega [12, Theorem 2.17].

Proposition 3.2. (Small data global existence I) Assume $p \geq 5$ and $\phi \in$ $\dot{H}^{s_{p}}(\mathbb{R})$. Then for any $A>0$ satisfying $\|h\|_{L^{\infty}} \leq A$ there exists $\varepsilon=\varepsilon(A)$ such that 
(i) There exists $B>0$ such that if $\phi$ satisfies $\|S(t) \phi\|_{L_{x}^{5(p-1) / 4} L_{t}^{5(p-1) / 2}} \leq \varepsilon$, then there exists a unique global solution $u$ to (3.1) such that

$$
\begin{aligned}
\|u\|_{L_{x}^{\frac{5}{4}(p-1)}} L_{t}^{\frac{5}{2}(p-1)} & \leq 2\|S(t) \phi\|_{L_{x}^{\frac{5}{4}(p-1)}} L_{t}^{\frac{5}{2}(p-1)} \\
\|u\|_{X_{\infty}} & \leq B\|\phi\|_{\dot{H}^{s}(\mathbb{R})}
\end{aligned}
$$

where $X_{\infty}$ is given by (1.6).

(ii) If $u$ is a global solution to (3.1) and satisfies $\|u\|_{L_{x}^{5(p-1) / 4} L_{t}^{5(p-1) / 2}} \leq 2 \varepsilon$, then

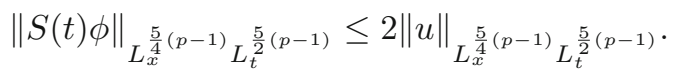

Proof. We first prove (i). By Lemma 3.1, there exists a unique solution $u \in$ $C\left([0, T(\phi, h)) ; \dot{H}^{s_{k}}(\mathbb{R})\right)$ to $(3.1)$. Let $u \in C\left(\left[0, T_{\max }\right) ; \dot{H}^{s_{k}}(\mathbb{R})\right)$ be a maximal solution to (3.1). Then $u$ satisfies

$$
\begin{aligned}
u(t) & =S(t) \phi-\int_{0}^{t} S\left(t-t^{\prime}\right) h\left(t^{\prime}\right) \partial_{x}\left(|u|^{p-1} u\right)\left(t^{\prime}\right) d t^{\prime} \\
& =: S(t) \phi+w(t) .
\end{aligned}
$$

By Propositions 2.5 and 2.8, we obtain

$$
\|w\|_{L_{x}^{\frac{5}{4}(p-1)} L_{T}^{\frac{5}{2}(p-1)}} \leq C A\|u\|_{L_{x}^{\frac{5}{4}(p-1)}}^{p-1} L_{T}^{\frac{5}{2}(p-1)}\left\|\left|\partial_{x}\right|^{s_{p}} u\right\|_{L_{x}^{5} L_{T}^{10}}
$$

for any $0<T<T_{\max }$. Since by Lemma 3.1 we have $\left\|\left|\partial_{x}\right|^{s_{p}} u\right\|_{L_{x}^{5} L_{T}^{10}}<\infty$, we see

$$
\|w\|_{L_{x}^{\frac{5}{4}(p-1)} L_{T}^{\frac{5}{2}(p-1)}} \leq C A\|u\|_{L_{x}^{\frac{5}{4}(p-1)} L_{T}^{\frac{5}{2}(p-1)}}^{p-1} .
$$

Combining (3.4) and $\|S(t) \phi\|_{L_{x}^{5(p-1) / 4} L_{t}^{5(p-1) / 2}} \leq \varepsilon$, we have

$$
\|u\|_{L_{x}^{\frac{5}{4}(p-1)} L_{T}^{\frac{5}{2}(p-1)}} \leq \varepsilon+C A\|u\|_{L_{x}^{\frac{5}{4}(p-1)} L_{T}^{\frac{5}{2}(p-1)}}^{p-1} .
$$

We now choose $\varepsilon=\varepsilon(A)$ so that $C A(2 \varepsilon)^{p-2}<1 / 2$ and $\varepsilon<1 / 2$. Then by the continuity of the norm, we have

$$
\|u\|_{L_{x}^{\frac{5}{4}(p-1)} L_{T_{\max }}^{\frac{5}{2}(p-1)}} \leq 2 \varepsilon .
$$

Therefore

$$
\begin{aligned}
\|u\|_{L_{x}^{\frac{5}{4}(p-1)} L_{T_{\max }}^{\frac{5}{2}(p-1)}} & \leq\|S(t) \phi\|_{L_{x}^{\frac{5}{4}(p-1)}} L_{T_{\max }^{\frac{5}{2}(p-1)}}+C A\|u\|^{p-1} L_{x}^{\frac{5}{4}(p-1)} L_{T_{\max }^{\frac{5}{2}(p-1)}} \\
& \leq\|S(t) \phi\|_{L_{x}^{\frac{5}{4}(p-1)} L_{T_{\max }}^{\frac{5}{2}(p-1)}}+C A(2 \varepsilon)^{p-2}\|u\|_{L_{x}^{\frac{5}{4}(p-1)}} L_{T_{\max }^{\frac{5}{4}(p-1)}} .
\end{aligned}
$$

Since $C A(2 \varepsilon)^{p-2}<1 / 2$, we obtain

$$
\|u\|_{L_{x}^{\frac{5}{4}(p-1)}} L_{T_{\max }}^{\frac{5}{2}(p-1)} \leq 2\|S(t) \phi\|_{L_{x}^{\frac{5}{4}(p-1)}} L_{T_{\max }}^{\frac{5}{2}(p-1)} .
$$


Propositions 2.5 and 2.8 imply

$$
\begin{aligned}
\|u\|_{X_{T}} & \leq C\|\phi\|_{\dot{H}^{s} p(\mathbb{R})}+C A\|u\|_{L_{x}^{\frac{5}{4}(p-1)} L_{T}^{\frac{5}{2}(p-1)}}^{p-1}\|u\|_{X_{T}} \\
& \leq C\|\phi\|_{\dot{H}^{s_{p}(\mathbb{R})}}+C A(2 \varepsilon)^{p-1}\|u\|_{X_{T}} \\
& \leq C\|\phi\|_{\dot{H}^{s_{p}(\mathbb{R})}}+\frac{1}{2}\|u\|_{X_{T}} .
\end{aligned}
$$

Letting $T \rightarrow T_{\max }$, we obtain

$$
\|u\|_{X_{T_{\max }} \leq 2 C\|\phi\|_{\dot{H}^{s} p(\mathbb{R})}} .
$$

In particular, we have

$$
\|u\|_{L_{x}^{\frac{5}{4}(p-1)} L_{T_{\max }}^{\frac{5}{2}(p-1)}} \leq 2 C\|\phi\|_{\dot{H}^{s} p(\mathbb{R})} .
$$

Hence by the blow up criterion (Lemma 3.1), we see $T_{\max }=\infty$. Combining this with (3.5) and (3.6), we obtain (i).

Next we prove (ii). Inequality (3.4) implies

$$
\begin{aligned}
& \|S(t) \phi\|_{L_{x}^{\frac{5}{4}(p-1)} L_{t}^{\frac{5}{2}(p-1)}} \leq\|u\|_{L_{x}^{\frac{5}{4}(p-1)} L_{t}^{\frac{5}{2}(p-1)}}+\|w\|_{L_{x}^{\frac{5}{4}(p-1)} L_{t}^{\frac{5}{2}(p-1)}} \\
& \leq\|u\|_{L_{x}^{\frac{5}{4}(p-1)} L_{t}^{\frac{5}{2}(p-1)}}+C A\|u\|_{L_{x}^{\frac{5}{4}(p-1)} L_{t}^{\frac{5}{2}(p-1)}}^{p-1} \\
& \leq\|u\|_{L_{x}^{\frac{5}{4}(p-1)} L_{t}^{\frac{5}{2}(p-1)}}\left(1+C A(2 \varepsilon)^{p-2}\right) .
\end{aligned}
$$

Since $C A(2 \varepsilon)^{p-2}<1$, we see

$$
\|S(t) \phi\|_{L_{x}^{\frac{5}{4}(p-1)} L_{t}^{\frac{5}{2}(p-1)}} \leq 2\|u\|_{L_{x}^{\frac{5}{4}(p-1)} L_{t}^{\frac{5}{2}(p-1)}} .
$$

Hence we have (ii). This completes the proof of Proposition 3.2.

Proposition 3.3. (Small data global existence II) Assume $p \geq 5$ and $\phi \in$ $\dot{H}^{s_{p}}(\mathbb{R})$. Let $h \in L^{\infty}(\mathbb{R})$ satisfy $\|h\|_{L^{\infty}} \leq A$. Let $u$ be a solution to (3.1) on maximal interval $\left[0, T_{\max }\right)$. Suppose further that for $\varepsilon=\varepsilon(A)>0$ and $B>0$ given in Lemma 3.2, there exists $T \in\left(0, T_{\max }\right)$ such that if $u$ satisfies $\|S(t) u(T)\|_{L_{x}^{5(p-1) / 4} L_{t}^{5(p-1) / 2}} \leq \varepsilon$. Then $T_{\max }=\infty$ and $u$ satisfies

$$
\begin{aligned}
\|u\|_{L_{x}^{\frac{5}{4}(p-1)}} L_{(T, \infty)}^{\frac{5}{2}(p-1)} & \leq 2 \varepsilon, \\
\|u\|_{X_{(T, \infty)}} & \leq B\|u(T)\|_{\dot{H}^{s} p(\mathbb{R})} .
\end{aligned}
$$

Proof. Let $T$ be given in the statement of the proposition and let $v$ be a solution to

$$
\begin{cases}\partial_{t} v+\partial_{x}^{3} v+h(t+T) \partial_{x}\left(|v|^{p-1} v\right)=0, & (t, x) \in \mathbb{R} \times \mathbb{R} \\ v(0, x)=u(T, x), & x \in \mathbb{R}\end{cases}
$$

Then $v$ can be rewritten as the integral equation

$$
v(t)=S(t) u(T)-\int_{0}^{t} S\left(t-t^{\prime}\right) h\left(t^{\prime}+T\right) \partial_{x}\left(|v|^{p-1} v\right)\left(t^{\prime}\right) d t^{\prime} .
$$

Since

$$
\|S(t) v(0)\|_{L_{x}^{\frac{5}{4}(p-1)} L_{t}^{\frac{5}{2}(p-1)}}=\|S(t) u(T)\|_{L_{x}^{\frac{5}{4}(p-1)} L_{t}^{\frac{5}{2}(p-1)}} \leq \varepsilon,
$$


Proposition 3.2 yields that $v$ exists globally and satisfies

$$
\begin{gathered}
\|v\|_{L_{x}^{\frac{5}{4}(p-1)} L_{t}^{\frac{5}{2}(p-1)}} \leq 2\|S(t) u(T)\|_{L_{x}^{\frac{5}{4}(p-1)} L_{t}^{\frac{5}{2}(p-1)}} \leq 2 \varepsilon, \\
\|v\|_{X_{\infty}} \leq B\|u(T)\|_{\dot{H}^{s}(\mathbb{R})} .
\end{gathered}
$$

Define $\tilde{u}$ by

$$
\tilde{u}(t):= \begin{cases}u(t) & (0 \leq t<T) \\ v(t-T) & (T \leq t<\infty)\end{cases}
$$

Then we see that $\tilde{u}$ satisfies the initial value problem $(3.1)$ on $[0, \infty)$. By the uniqueness of (3.1) (Lemma 3.1), we see that $\tilde{u}=u$ and $T_{\max }=\infty$. The inequalities (3.9) and (3.10) imply

$$
\begin{aligned}
\|u\|_{L_{x}^{\frac{5}{4}(p-1)} L_{(T, \infty)}^{\frac{5}{2}(p-1)}} & =\|v\|_{L_{x}^{\frac{5}{4}(p-1)} L_{t}^{\frac{5}{2}(p-1)}} \leq 2 \varepsilon, \\
\|u\|_{X_{(T, \infty)}} & =\|v\|_{X_{t}} \leq B\|u(T)\|_{\dot{H}^{s} p(\mathbb{R})} .
\end{aligned}
$$

This completes the proof of Proposition 3.3.

Lemma 3.4. Let $p \geq 5$ and $T \in(0, \infty]$. Then for any $f$ satisfying $\left|\partial_{x}\right|^{s_{p}} f \in$ $L_{x}^{1} L_{T}^{2}$, we have

$$
\int_{0}^{t} g\left(\omega t^{\prime}\right) S\left(t-t^{\prime}\right) \partial_{x} f\left(t^{\prime}\right) d t^{\prime} \longrightarrow m(g) \int_{0}^{t} S\left(t-t^{\prime}\right) \partial_{x} f\left(t^{\prime}\right) d t^{\prime} \quad \text { in } \quad X_{T},
$$

as $|\omega| \rightarrow \infty$.

Proof. Since the proof is now standard, we omit the detail (see [3, Lemma 3.1] for instance).

Proposition 3.5. Suppose $p \geq 5$ and $\phi \in \dot{H}^{s_{p}}(\mathbb{R})$. Let $u_{\omega}$ be a maximal solution to (1.1) and let $U$ be a solution to $(1.5)$ on maximal interval $\left[0, S_{\max }\right)$. Assume that for any $T$ satisfying $0<T<S_{\max }, u_{\omega}$ exists on $[0, T)$ for $|\omega|$ large and

$$
\limsup _{|\omega| \rightarrow \infty}\left(\left\|\left|\partial_{x}\right|^{s_{p}} u_{\omega}\right\|_{L_{x}^{5} L_{T}^{10}}+\left\|u_{\omega}\right\|_{L_{x}^{\frac{5}{4}(p-1)} L_{T}^{\frac{5}{2}(p-1)}}\right)<\infty
$$

holds. Then we have

$$
\left\|u_{\omega}-U\right\|_{X_{T}} \longrightarrow 0 \quad \text { as } \quad|\omega| \rightarrow \infty
$$


Proof. We first choose $A<\infty$ satisfying $\|g\|_{L^{\infty}} \leq A$. Since $u_{\omega}$ and $U$ satisfy (1.1) and (1.5),

$$
\begin{aligned}
&\left(u_{\omega}-U\right)(t) \\
&=-\int_{0}^{t} g\left(\omega t^{\prime}\right) S\left(t-t^{\prime}\right) \partial_{x}\left(\left|u_{\omega}\right|^{p-1} u_{\omega}\right) d t^{\prime} \\
&+m(g) \int_{0}^{t} S\left(t-t^{\prime}\right) \partial_{x}\left(|U|^{p-1} U\right) d t^{\prime} \\
&=-\int_{0}^{t} g\left(\omega t^{\prime}\right) S\left(t-t^{\prime}\right) \partial_{x}\left(\left|u_{\omega}\right|^{p-1} u_{\omega}-|U|^{p-1} U\right) d t^{\prime} \\
&-\int_{0}^{t}\left(g\left(\omega t^{\prime}\right)-m(g)\right) S\left(t-t^{\prime}\right) \partial_{x}\left(|U|^{p-1} U\right) d t^{\prime} \\
&=: I_{1}+I_{2} .
\end{aligned}
$$

We show $\left\|I_{2}\right\|_{X_{T}} \rightarrow$ 0. By Proposition 2.8 and Lemma 3.1,

$$
\left\|\left|\partial_{x}\right|^{s_{p}}\left(|U|^{p-1} U\right)\right\|_{L_{x}^{1} L_{T}^{2}} \leq C\|U\|_{L_{x}^{\frac{5}{4}(p-1)} L_{T}^{\frac{5}{2}(p-1)}}^{p-1}\left\|\left|\partial_{x}\right|^{s_{p}} U\right\|_{L_{x}^{5} L_{T}^{10}}<\infty .
$$

Hence by Lemma 3.4,

$$
\left\|I_{2}\right\|_{X_{T}}=: C_{\omega} \longrightarrow 0 \text { as }|\omega| \rightarrow \infty .
$$

Next, we evaluate $I_{1}$. Propositions 2.5 and 2.8 imply

$$
\begin{aligned}
& \left\|I_{1}\right\|_{X_{T}} \\
& \leq C A\left\|\left|\partial_{x}\right|^{s_{p}}\left(\left|u_{\omega}\right|^{p-1} u_{\omega}-|U|^{p-1} U\right)\right\|_{L_{x}^{1} L_{T}^{2}} \\
& \leq C A\left\{\left(\left\|u_{\omega}\right\|_{L_{x}^{\frac{5}{4}(p-1)} L_{T}^{\frac{5}{2}(p-1)}}^{p-1}+\|U\|_{L_{x}^{\frac{5}{4}(p-1)} L_{T}^{\frac{5}{2}(p-1)}}^{p-1}\right)\left\|\left|\partial_{x}\right|^{s_{p}}\left(u_{\omega}-U\right)\right\|_{L_{x}^{5} L_{T}^{10}}\right. \\
& +\left(\left\|u_{\omega}\right\|_{L_{x}^{\frac{5}{4}(p-1)}}^{p-2} L_{T}^{\frac{5}{2}(p-1)}+\|U\|_{L_{x}^{\frac{5}{4}(p-1)}}^{p-2} L_{T}^{\frac{5}{2}(p-1)}\right) \\
& \left.\times\left(\left\|\left|\partial_{x}\right|^{s_{p}} u_{\omega}\right\|_{L_{x}^{5} L_{T}^{10}}+\left\|\left|\partial_{x}\right|^{s_{p}} U\right\|_{L_{x}^{5} L_{T}^{10}}\right)\left\|u_{\omega}-U\right\|_{L_{x}^{\frac{5}{4}(p-1)} L_{T}^{\frac{5}{2}(p-1)}}\right\} .
\end{aligned}
$$

Set

$$
\begin{aligned}
M_{T}= & \left\|\left|\partial_{x}\right|^{s_{p}} u_{\omega}\right\|_{L_{x}^{5} L_{T}^{10}}+\left\|\left|\partial_{x}\right|^{s_{p}} U\right\|_{L_{x}^{5} L_{T}^{10}}+\left\|u_{\omega}\right\|_{L_{x}^{\frac{5}{4}(p-1)}} L_{T}^{\frac{5}{2}(p-1)} \\
& +\|U\|_{L_{x}^{\frac{5}{4}(p-1)} L_{T}^{5(p-1) / 2}} .
\end{aligned}
$$

Then we have

$$
\left\|I_{1}\right\|_{X_{T}} \leq C A M_{T}^{p-1}\left\|u_{\omega}-U\right\|_{X_{T}} .
$$

We split the time interval $[0, T]$ into subintervals $\left[t_{i}, t_{i+1}\right], i=0, \ldots, J-1$ and $t_{0}=0, t_{J}=T$ so that for each intervals $\left[t_{i}, t_{i+1}\right]$,

$$
C A M_{\left[t_{i}, t_{i+1}\right]}^{p-1} \leq \frac{1}{2}
$$


hold, where $C$ is the constant in (3.18), and

$$
\begin{aligned}
M_{\left[t_{i}, t_{i+1}\right]}= & \left\|\left|\partial_{x}\right|^{s_{p}} u_{\omega}\right\|_{L_{x}^{5} L_{\left[t_{i}, t_{i+1}\right]}^{10}}+\left\|\left|\partial_{x}\right|^{s_{p}} U\right\|_{L_{x}^{5} L_{\left[t_{i}, t_{i+1}\right]}^{10}}+\|U\|_{L_{x}^{\frac{5}{4}(p-1)}} L_{\left[t_{i}, t_{i+1}\right]}^{\frac{5}{2}(p-1)} . \\
& +\left\|u_{\omega}\right\|_{L_{x}^{\frac{5}{4}(p-1)} L_{\left[t_{i}, t_{i+1}\right]}^{\frac{5}{2}(p-1)}}+
\end{aligned}
$$

In Appendix, we show the existence of a subdivision satisfying (3.19). From (1.1) and (1.5),

$$
\begin{aligned}
& \left(u_{\omega}-U\right)(t) \\
& =S\left(t-t_{i}\right)\left(u_{\omega}\left(t_{i}\right)-U\left(t_{i}\right)\right) \\
& \quad-\int_{t_{i}}^{t} g\left(\omega t^{\prime}\right) S\left(t-t^{\prime}\right) \partial_{x}\left(\left|u_{\omega}\right|^{p-1} u_{\omega}-|U|^{p-1} U\right) d t^{\prime} \\
& \quad-\int_{t_{i}}^{t}\left(g\left(\omega t^{\prime}\right)-m(g)\right) S\left(t-t^{\prime}\right) \partial_{x}\left(|U|^{p-1} U\right) d t^{\prime} \\
& =: S\left(t-t_{i}\right)\left(u_{\omega}\left(t_{i}\right)-U\left(t_{i}\right)\right)+I_{i, 1}+I_{i, 2} .
\end{aligned}
$$

For the first term on the right hand side of (3.21), we apply Proposition 2.5 to obtain

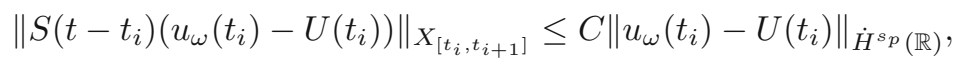

where $\|f\|_{X_{\left[t_{i}, t_{i+1}\right]}}=\|f\|_{L_{\left[t_{i}, t_{i+1}\right]}^{\infty} \dot{H}^{s_{p}}}+\left\|\left|\partial_{x}\right|^{s_{p}} f\right\|_{L_{x}^{5} L_{\left[t_{i}, t_{i+1}\right]}^{10}}+\|f\|_{L_{x}^{\frac{5}{4}(p-1)} L_{\left[t_{i}, t_{i+1}\right]}^{\frac{5}{2}(p-1)}}$. Propositions 2.5 and 2.8 imply

$$
\begin{aligned}
& \left\|I_{i, 1}\right\|_{X_{\left[t_{i}, t_{i+1}\right]}} \leq C A\left\{\left(\left\|u_{\omega}\right\|_{L_{x}^{\frac{5}{4}(p-1)} L_{\left[t_{i}, t_{i+1}\right]}^{\frac{5}{2}(p-1)}}^{p-1}+\|U\|_{L_{x}^{\frac{5}{4}(p-1)} L_{\left[t_{i}, t_{i+1}\right]}^{\frac{5}{2}(p-1)}}^{p-1}\right)\right. \\
& \times\left.|| \partial_{x}\right|^{s_{p}}\left(u_{\omega}-U\right) \|_{L_{x}^{5} L_{\left[t_{i}, t_{i+1}\right]}^{10}}
\end{aligned}
$$

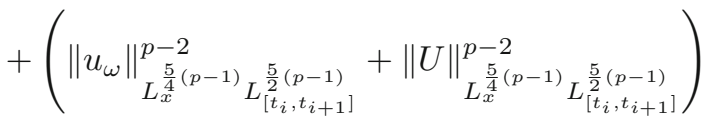

$$
\begin{aligned}
& \times\left(\left.|| \partial_{x}\right|^{s_{p}} u_{\omega}\left\|_{L_{x}^{5} L_{\left[t_{i}, t_{i+1}\right]}^{10}}+\right\|\left|\partial_{x}\right|^{s_{p}} U \|_{L_{x}^{5} L_{\left[t_{i}, t_{i+1}\right]}^{10}}\right) \\
& \left.\times\left\|u_{\omega}-U\right\|_{L_{x}^{\frac{5}{4}(p-1)} L_{\left[t_{i}, t_{i+1}\right]}^{\frac{5}{2}(p-1)}}\right\} . \\
& \leq C A M_{\left[t_{i}, t_{i+1}\right]}^{p-1}\left\|u_{\omega}-U\right\|_{X_{\left[t_{i}, t_{i+1}\right]}} \\
& \leq \frac{1}{2}\left\|u_{\omega}-U\right\|_{X_{\left[t_{i}, t_{i+1}\right]}} \text {. }
\end{aligned}
$$

Combining (3.16), (3.21), (3.22) and (3.23), we have

$$
\left\|u_{\omega}-U\right\|_{X_{\left[t_{i}, t_{i+1}\right]}} \leq C\left\|u_{\omega}\left(t_{i}\right)-U\left(t_{i}\right)\right\|_{\dot{H}^{s} p(\mathbb{R})}+\frac{1}{2}\left\|u_{\omega}-U\right\|_{X_{\left[t_{i}, t_{i+1}\right]}}+C_{\omega},
$$

which implies

$$
\left\|u_{\omega}-U\right\|_{X_{\left[t_{i}, t_{i+1}\right]}} \leq 2 C\left\|u_{\omega}\left(t_{i}\right)-U\left(t_{i}\right)\right\|_{\dot{H}^{s_{p}}(\mathbb{R})}+2 C_{\omega} .
$$


Noting $u_{\omega}(0)-U(0)=0$, we see from (3.24) with $i=0$,

$$
\left\|u_{\omega}-U\right\|_{X_{\left[0, t_{1}\right]}} \leq 2 C_{\omega} .
$$

In particular,

$$
\left\|u_{\omega}\left(t_{1}\right)-U\left(t_{1}\right)\right\|_{\dot{H}^{s_{p}(\mathbb{R})}} \leq 2 C_{\omega} .
$$

By (3.24) with $i=1$ and (3.25), we find

$$
\left\|u_{\omega}-U\right\|_{X_{\left[t_{1}, t_{2}\right]}} \leq 2 C_{\omega}+4 C C_{\omega} .
$$

In particular, we obtain

$$
\left\|u_{\omega}\left(t_{2}\right)-U\left(t_{2}\right)\right\|_{\dot{H}^{s} p(\mathbb{R})} \leq 2 C_{\omega}+4 C C_{\omega} .
$$

Repeating this argument, we have

$$
\left\|u_{\omega}-U\right\|_{X_{T}} \leq \sum_{i=0}^{J-1}\left\|u_{\omega}-U\right\|_{X_{\left[t_{i}, t_{i+1}\right]}} \leq 2 \frac{(2 C)^{J}-1}{2 C-1} C_{\omega} .
$$

By Appendix A, we see that $J \leq(4 C A)^{5 / 2} M_{T}^{5(p-1) / 2}$. Hence we have (3.13).

Proposition 3.6. Assume $p \geq 5$ and $\phi \in \dot{H}^{s_{p}}(\mathbb{R})$. Let $u_{\omega}$ be a maximal solution to (1.1) and let $U$ be a solution to (1.5) on maximal interval $\left[0, S_{\max }\right)$. Then for any $T \in\left(0, S_{\max }\right)$, $u_{\omega}$ exists on $[0, T)$ for $|\omega|$ large. Furthermore,

$$
\left\|u_{\omega}-U\right\|_{X_{T}} \longrightarrow 0 \text { as }|\omega| \rightarrow \infty \text {. }
$$

Proof. Let $A=\|g\|_{L^{\infty}}$ and fix $T \in\left(0, S_{\max }\right)$. We split the time interval $[0, T]$ into subintervals $\left[t_{i}, t_{i+1}\right], i=0, \ldots, J-1$ and $t_{0}=0, t_{J}=T$, where $t_{i}$ are fixed later. For the interval $\left[t_{i}, t_{i+1}\right]$, we define $M_{\left[t_{i}, t_{i+1}\right]}^{\omega}$ by

$$
M_{\left[t_{i}, t_{i+1}\right]}^{\omega}=\left\|\left|\partial_{x}\right|^{s_{p}} u_{\omega}\right\|_{L_{x}^{5} L_{\left[t_{i}, t_{i+1}\right]}^{10}}+\left\|u_{\omega}\right\|_{L_{x}^{\frac{5}{4}(p-1)} L_{\left[t_{i}, t_{i+1}\right]}^{\frac{5}{2}(p-1)}} .
$$

By the Duhamel formula,

$$
u_{\omega}(t)=S\left(t-t_{i}\right) u_{\omega}\left(t_{i}\right)-\int_{t_{i}}^{t} g\left(\omega t^{\prime}\right) S\left(t-t^{\prime}\right) \partial_{x}\left(\left|u_{\omega}\right|^{p-1} u_{\omega}\right)\left(t^{\prime}\right) d t^{\prime}
$$

for $t \in\left[t_{i}, t_{i+1}\right]$. Propositions 2.5 and 2.8 yield

$$
\begin{aligned}
& M_{\left[t_{i}, t_{i+1}\right]}^{\omega} \leq\left\|S\left(t-t_{i}\right)\left|\partial_{x}\right|^{s_{p}} u_{\omega}\left(t_{i}\right)\right\|_{L_{x}^{5} L_{\left[t_{i}, t_{i+1}\right]}^{10}}
\end{aligned}
$$

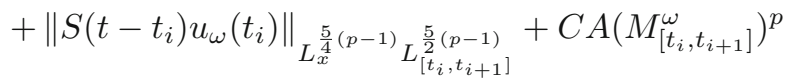

$$
\begin{aligned}
& \leq\left\|S\left(t-t_{i}\right)\left|\partial_{x}\right|^{s_{p}}\left(u_{\omega}\left(t_{i}\right)-U\left(t_{i}\right)\right)\right\|_{L_{x}^{5} L_{\left[t_{i}, t_{i+1}\right]}^{10}} \\
& +\left\|S\left(t-t_{i}\right)\left|\partial_{x}\right|^{s_{p}} U\left(t_{i}\right)\right\|_{L_{x}^{5} L_{\left[t_{i}, t_{i+1}\right]}^{10}} \\
& +\left\|S\left(t-t_{i}\right)\left(u_{\omega}\left(t_{i}\right)-U\left(t_{i}\right)\right)\right\|_{L_{x}^{\frac{5}{4}(p-1)}} L_{\left[t_{i}, t_{i+1}\right]}^{\frac{5}{2}(p-1)} \\
& +\left\|S\left(t-t_{i}\right) U\left(t_{i}\right)\right\|_{L_{x}^{\frac{5}{4}(p-1)} L_{\left[t_{i}, t_{i+1}\right]}^{\frac{5}{2}(p-1)}}+C A\left(M_{\left[t_{i}, t_{i+1}\right]}^{\omega}\right)^{p} .
\end{aligned}
$$

We now choose $t_{i}$ so that for each $i$, the inequalities

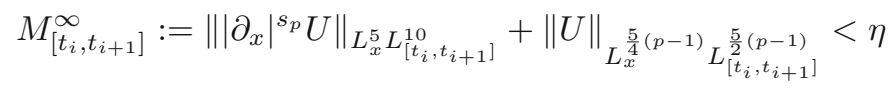


hold, where $\eta>0$ is fixed later. The same argument as that in Appendix A yields that $J \leq C \eta^{-5(p-1) / 2}\left(M_{S_{\max }}^{\infty}\right)^{5(p-1) / 2}$, where $M_{T}^{\infty}:=\left\|\left|\partial_{x}\right|^{s_{p}} U\right\|_{L_{x}^{5} L_{T}^{10}}+$ $\|U\|_{L_{x}^{5(p-1) / 4} L_{T}^{5(p-1) / 2} \text {. }}$

By an argument similar to the proof of (3.29),

$$
\begin{aligned}
& \left\|S\left(t-t_{i}\right)\left|\partial_{x}\right|^{s_{p}} U\left(t_{i}\right)\right\|_{L_{x}^{5} L_{\left[t_{i}, t_{i+1}\right]}^{10}}+\left\|S\left(t-t_{i}\right) U\left(t_{i}\right)\right\|_{L_{x}^{\frac{5}{4}(p-1)}} L_{\left[t_{i}, t_{i+1}\right]}^{\frac{5}{2}(p-1)} \\
& \quad \leq M_{\left[t_{i}, t_{i+1}\right]}^{\infty}+C A\left(M_{\left[t_{i}, t_{i+1}\right]}^{\infty}\right)^{p} \leq \eta+C A \eta^{p} .
\end{aligned}
$$

We choose $\varepsilon>0$ so that $C A \eta^{p-1}<1$. Then we see that the right hand side of (3.31) is bounded by $2 \eta$. Further, choosing $\eta$ so small that $C A(6 \eta)^{p-1}<1 / 2$. Then we obtain

$C A\left\{2\left(\left\|S\left(t-t_{i}\right)\left|\partial_{x}\right|^{s_{p}} U\left(t_{i}\right)\right\|_{L_{x}^{5} L_{\left[t_{i}, t_{i+1}\right]}^{10}}+\left\|S\left(t-t_{i}\right) U\left(t_{i}\right)\right\|_{L_{x}^{\frac{5}{4}(p-1)} L_{\left[t_{i}, t_{i+1}\right]}^{\frac{5}{2}(p-1)}}+\eta\right)\right\}^{p-1}<\frac{1}{2}$.

Noting $u_{\omega}\left(t_{0}\right)-U\left(t_{0}\right)=0$, we find

$$
M_{\left[0, t_{1}\right]}^{\omega} \leq\left\|\left|\partial_{x}\right|^{s_{p}} S(t) \phi\right\|_{L_{x}^{5} L_{\left[0, t_{1}\right]}^{10}}+\|S(t) \phi\|_{L_{x}^{\frac{5}{4}(p-1)} L_{\left[0, t_{1}\right]}^{\frac{5}{2}(p-1)}}+C A\left(M_{\left[0, t_{1}\right]}^{\omega}\right)^{p} .
$$

By (3.32),

$$
C A\left\{2\left(\left\|\left|\partial_{x}\right|^{s_{p}} S(t) \phi\right\|_{L_{x}^{5} L_{\left[0, t_{1}\right]}^{10}}+\|S(t) \phi\|_{L_{x}^{\frac{5}{4}(p-1)} L_{\left[0, t_{1}\right]}^{\frac{5}{2}(p-1)}}\right)\right\}^{p-1}<\frac{1}{2} .
$$

By (3.33), (3.34) and the continuity of the norm, we have

$$
M_{\left[0, t_{1}\right]}^{\omega} \leq 2\left(\left\|\left|\partial_{x}\right|^{s p} S(t) \phi\right\|_{L_{x}^{5} L_{\left[0, t_{1}\right]}^{10}}+\|S(t) \phi\|_{L_{x}^{\frac{5}{4}(p-1)} L_{\left[0, t_{1}\right]}^{\frac{5}{2}(p-1)}}\right) \leq C\|\phi\|_{\dot{H}^{s_{p}}(\mathbb{R})} .
$$

We show $T_{\max }>t_{1}$ by contradiction argument. We assume that $T_{\max } \leq t_{1}$. Then by (3.35),

$$
\left\|u_{\omega}\right\|_{L_{x}^{\frac{5}{4}(p-1)}} L_{T_{\max }}^{\frac{5}{2}(p-1)} \leq M_{\left[0, t_{1}\right]}^{\omega} \leq C\|\phi\|_{\dot{H}^{s p}(\mathbb{R})} .
$$

Hence by the blow up criterion for (1.1) (Lemma 3.1), we see $T_{\max }=\infty$ which contradicts $T_{\max } \leq t_{1}$. Hence $T_{\max }>t_{1}$. Furthermore, by Proposition 3.5, we obtain

$$
\left\|u_{\omega}-U\right\|_{X_{\left[0, t_{1}\right]}} \longrightarrow 0 \text { as }|\omega| \rightarrow \infty .
$$

Next we consider the case $i=1$. By Proposition 2.5,

$$
\begin{aligned}
& \left\|S\left(t-t_{1}\right)\left|\partial_{x}\right|^{s_{p}}\left(u_{\omega}\left(t_{1}\right)-U\left(t_{1}\right)\right)\right\|_{L_{x}^{5} L_{\left[t_{1}, t_{2}\right]}^{10}} \\
& \quad+\left\|S\left(t-t_{1}\right)\left(u_{\omega}\left(t_{1}\right)-U\left(t_{1}\right)\right)\right\|_{L_{x}^{\frac{5}{4}(p-1)} L_{\left[t_{1}, t_{2}\right]}^{\frac{5}{2}(p-1)}} \\
& \quad \leq\left\|u_{\omega}\left(t_{1}\right)-U\left(t_{1}\right)\right\|_{\dot{H}^{s p}(\mathbb{R})} \\
& \quad \leq\left\|u_{\omega}-U\right\|_{X_{\left[t_{0}, t_{1}\right]}} .
\end{aligned}
$$


By (3.29) with $i=1,(3.36)$ and (3.37), for $|\omega|$ sufficiently large,

$$
\begin{aligned}
M_{\left[t_{1}, t_{2}\right]}^{\omega} \leq & \left\|S\left(t-t_{1}\right)\left|\partial_{x}\right|^{s_{p}} U\left(t_{1}\right)\right\|_{L_{x}^{5} L_{\left[t_{1}, t_{2}\right]}^{10}} \\
& +\left\|S\left(t-t_{1}\right) U\left(t_{1}\right)\right\|_{L_{x}^{\frac{5}{4}(p-1)}} L_{\left[t_{1}, t_{2}\right]}^{\frac{5}{2}(p-1)}+C A\left(M_{\left[t_{1}, t_{2}\right]}^{\omega}\right)^{p}+C_{\omega},
\end{aligned}
$$

where $C_{\omega}$ satisfies $C_{\omega} \rightarrow 0$ as $|\omega| \rightarrow \infty$. On the other hand by (3.32) with $i=1$,

$C A\left\{2\left(\left\|S\left(t-t_{1}\right)\left|\partial_{x}\right|^{s_{p}} U\left(t_{1}\right)\right\|_{L_{x}^{5} L_{\left[t_{1}, t_{2}\right]}^{10}}+\left\|S\left(t-t_{1}\right) U\left(t_{1}\right)\right\|_{L_{x}^{\frac{5}{4}(p-1)} L_{\left[t_{1}, t_{2}\right]}^{\frac{5}{2}(p-1)}}+C_{\omega}\right)\right\}^{p-1}<\frac{1}{2}$

Hence by an argument similar to that in the case $i=0$, we see that $u_{\omega}$ exists on $\left[t_{0}, t_{2}\right]$ for $|\omega|$ large, and

$$
\left\|u_{\omega}-U\right\|_{X_{\left[t_{1}, t_{2}\right]}} \longrightarrow 0 \quad \text { as } \quad|\omega| \rightarrow \infty .
$$

Since $J$ is finite, we can repeat this argument $J$ times. Hence we have that for $|\omega|$ sufficiently large, $u_{\omega}$ exists on $[0, T]$ and satisfies (3.26). This completes the proof of Proposition 3.6.

\section{Proof of Theorem $\mathbf{1 . 2}$}

In this section, we prove Theorem 1.2. Let $\varepsilon_{0}=\varepsilon(A)$ be given in Proposition 3.2 and let $\varepsilon \in\left(0, \varepsilon_{0}\right)$. By the assumption $\|U\|_{L_{x}^{5(p-1) / 4} L_{t}^{5(p-1) / 2}}<\infty$, for $\varepsilon>0$, we can choose $T>0$ sufficiently large so that

$$
\|U\|_{L_{x}^{\frac{5}{4}(p-1)} L_{(T, \infty)}^{\frac{5}{2}(p-1)}} \leq \frac{\varepsilon}{4}
$$

holds. Let $\tilde{U}(t):=U(t+T)$. By (4.1),

$$
\|\tilde{U}\|_{L_{x}^{\frac{5}{4}(p-1)} L_{t}^{\frac{5}{2}(p-1)}}=\|U\|_{L_{x}^{\frac{5}{4}(p-1)} L_{(T, \infty)}^{\frac{5}{2}(p-1)}} \leq \frac{\varepsilon}{4} .
$$

Hence by Proposition 3.2 (ii),

$$
\begin{gathered}
\|S(t) U(T)\|_{L_{x}^{\frac{5}{4}(p-1)} L_{t}^{\frac{5}{2}(p-1)}}=\|S(t) \tilde{U}(0)\|_{L_{x}^{\frac{5}{4}(p-1)}} L_{t}^{\frac{5}{2}(p-1)} \\
\quad \leq 2\|\tilde{U}\|_{L_{x}^{\frac{5}{4}(p-1)} L_{t}^{\frac{5}{2}(p-1)}}=2\|U\|_{L_{x}^{\frac{5}{4}(p-1)} L_{(T, \infty)}^{\frac{5}{2}(p-1)}} \leq \frac{\varepsilon}{2} .
\end{gathered}
$$

Applying Proposition 3.3, we see

$$
\|U\|_{X_{(T, \infty)}} \leq B\|U(T)\|_{\dot{H}^{s p}(\mathbb{R})} .
$$

Proposition 3.6 yields

$$
\sup _{0<t \leq T}\left\|u_{\omega}(t)-U(t)\right\|_{\dot{H}^{s}(\mathbb{R})} \longrightarrow 0 \quad \text { as } \quad|\omega| \rightarrow \infty .
$$


By Proposition 2.5, (4.2) and (4.4), we find that if $|\omega|$ is sufficiently large, then

$$
\begin{aligned}
& \left\|S(t) u_{\omega}(T)\right\|_{L_{x}^{\frac{5}{4}(p-1)} L_{T}^{\frac{5}{2}(p-1)}} \\
& \quad \leq\left\|S(t) u_{\omega}(T)-S(t) U(T)\right\|_{L_{x}^{\frac{5}{4}(p-1)} L_{T}^{\frac{5}{2}(p-1)}+\|S(t) U(T)\|_{L_{x}^{\frac{5}{4}(p-1)}} L_{T}^{\frac{5}{2}(p-1)}} \\
& \quad \leq C\left\|u_{\omega}(T)-U(T)\right\|_{\dot{H}^{s} p(\mathbb{R})}+\frac{\varepsilon}{2} . \\
& \quad \leq \varepsilon .
\end{aligned}
$$

Hence by Proposition 3.3, we see that if $|\omega|$ is sufficiently large, then $u_{\omega}$ exists globally and

$$
\begin{aligned}
\left\|u_{\omega}\right\|_{L_{x}^{\frac{5}{4}(p-1)} L_{(T, \infty)}^{\frac{5}{2}(p-1)}} & \leq 2 \varepsilon \\
\left\|u_{\omega}\right\|_{X_{(T, \infty)}} & \leq B\left\|u_{\omega}(T)\right\|_{\dot{H}^{s_{p}}(\mathbb{R})} .
\end{aligned}
$$

Next we show (1.8). Set $M_{0}:=\sup _{0 \leq t \leq T}\|U(t)\|_{\dot{H}^{s_{p}(\mathbb{R})}}$. By (4.4) and (4.6), we have for $\omega_{0}>0$ sufficiently large,

$$
\begin{aligned}
& \sup _{|\omega| \geq \omega_{0}} \sup _{t \geq 0}\left\|u_{\omega}(t)\right\|_{\dot{H}^{s} p(\mathbb{R})} \\
& \leq \sup _{|\omega| \geq \omega_{0}} \sup _{t \geq T}\left\|u_{\omega}(t)\right\|_{\dot{H}^{s_{p}(\mathbb{R})}}+\sup _{|\omega| \geq \omega_{0}} \sup _{0 \leq t \leq T}\left\|u_{\omega}(t)-U(t)\right\|_{\dot{H}^{s} p(\mathbb{R})} \\
& \quad+\sup _{0 \leq t \leq T}\|U(t)\|_{\dot{H}^{s_{p}}(\mathbb{R})} \\
& \quad \leq B \sup _{|\omega| \geq \omega_{0}}\left\|u_{\omega}(T)\right\|_{\dot{H}^{s_{p}}(\mathbb{R})}+1+M_{0}=: M_{1}
\end{aligned}
$$

By the Duhamel formula,

$$
\begin{aligned}
u_{\omega}(T+t)-U(T+t)= & S(t)\left(u_{\omega}(T)-U(T)\right) \\
& -\int_{0}^{t} S\left(t-T-t^{\prime}\right) g\left(\omega\left(T+t^{\prime}\right)\right) \partial_{x}\left(\left|u_{\omega}\right|^{p-1} u_{\omega}\right)\left(T+t^{\prime}\right) d t^{\prime} \\
& +m(g) \int_{0}^{t} S\left(t-T-t^{\prime}\right) \partial_{x}\left(|U|^{p-1} U\right)\left(T+t^{\prime}\right) d t^{\prime} \\
= & : I_{1}+I_{2}+I_{3} .
\end{aligned}
$$

We evaluate the $X_{\infty}$ norm for $I_{i}, i=1,2,3$. For the term $I_{1}$, we apply Proposition 2.5 and (4.4) to conclude

$$
\left\|I_{1}\right\|_{X_{\infty}} \leq C\left\|u_{\omega}(T)-U(T)\right\|_{\dot{H}^{s p}(\mathbb{R})} \longrightarrow 0 \quad \text { as } \quad|\omega| \rightarrow \infty .
$$

By Propositions 2.5 and 2.8, and the inequality (4.6),

$$
\begin{aligned}
\left\|I_{2}\right\|_{X_{\infty}} & \leq C A\left\|u_{\omega}(T+\cdot)\right\|_{L_{x}^{\frac{5}{4}(p-1)} L_{t}^{\frac{5}{2}(p-1)}}^{p-1}\left\|u_{\omega}(T+\cdot)\right\|_{X_{\infty}} \\
& =C A\left\|u_{\omega}\right\|_{L_{x}^{\frac{5}{4}(p-1)}}^{p} L_{(T, \infty)}^{\frac{5}{2}(p-1)}\left\|u_{\omega}\right\|_{X_{(T, \infty)}} \\
& \leq C A(2 \varepsilon)^{p-1} B\left\|u_{\omega}(T)\right\|_{\dot{H}^{s_{p}}(\mathbb{R})} \\
& \leq C A(2 \varepsilon)^{p-1} B M_{1} .
\end{aligned}
$$


In a similar way, by Propositions 2.5 and 2.8 and the inequalities (4.1), (4.3) and (4.6),

$$
\begin{aligned}
\left\|I_{3}\right\|_{X_{\infty}} & \leq C A\|U(T+\cdot)\|_{L_{x}^{\frac{5}{4}(p-1)} L_{t}^{\frac{5}{2}(p-1)}}^{p-1}\|U(T+\cdot)\|_{X_{\infty}} \\
& =C A\|U\|_{L_{x}^{\frac{5}{4}(p-1)}}^{p-1} L_{(T, \infty)}^{\frac{5}{2}(p-1)}\|U\|_{X_{(T, \infty)}} \\
& \leq C A\left(\frac{\varepsilon}{4}\right)^{p-1} B\|U(T)\|_{\dot{H}^{s} p(\mathbb{R})} \\
& \leq C A\left(\frac{\varepsilon}{4}\right)^{p-1} B M_{0} .
\end{aligned}
$$

Let $\delta>0$ be an arbitrary number. Then by (4.9), for $|\omega|$ sufficiently large, we have

$$
\left\|I_{1}\right\|_{X_{\infty}}<\frac{\delta}{2}
$$

Furthermore, we choose $\varepsilon>0$ so that $C A B \varepsilon^{p-1}\left(M_{0}+M_{1}\right)<\delta / 2$. Then by (4.8), (4.10), (4.11) and (4.12),

$$
\begin{aligned}
\left\|u_{\omega}-U\right\|_{X_{(T, \infty)}} & =\left\|u_{\omega}(T+\cdot)-U(T+\cdot)\right\|_{X_{\infty}} \\
& \leq\left\|I_{1}\right\|_{X_{\infty}}+\left\|I_{2}\right\|_{X_{\infty}}+\left\|I_{3}\right\|_{X_{\infty}}<\delta .
\end{aligned}
$$

On the other hand, by Proposition 3.6,

$$
\left\|u_{\omega}-U\right\|_{X_{T}} \longrightarrow 0 \text { as }|\omega| \rightarrow \infty .
$$

Collecting (4.13) and (4.14), we obtain (1.8). This completes the proof of Theorem 1.2 .

\section{Subdivision of time interval}

In this section, we show that for any $T \in(0, \infty)$, there exists a positive integer $J$ satisfying $J \leq(4 C A)^{5 / 2} M_{T}^{5(p-1) / 2}$ and a sequence $0=t_{0}<t_{1}<\cdots<t_{J}=$ $T$ such that $[0, T]=\bigcup_{i=0}^{J-1} I_{j}, I_{j}=\left[t_{i}, t_{i+1}\right]$ and

$$
\frac{1}{4} \leq C A M_{\left[t_{i}, t_{i+1}\right]}^{p-1} \leq \frac{1}{2} \quad \text { for any } \quad 0 \leq i \leq J-1
$$

where $C$ is the constant in (3.19) and $M_{\left[t_{i}, t_{i+1}\right]}$ is defined by (3.20). We may assume $C A M_{[0, T]}^{p-1}>1 / 2$ unless there is nothing to prove.

We first choose $t_{1} \in[0, T]$ so that $t_{0}<t_{1}$ and $C A M_{\left[t_{0}, t_{1}\right]}^{p-1}=1 / 2$. Similarly, if $C A M_{\left[t_{i}, T\right]}^{p-1}>1 / 2$, then we choose $t_{i+1} \in[0, T]$ so that $t_{i}<t_{i+1}$ and $C A M_{\left[t_{i}, t_{i+1}\right]}^{p-1}=1 / 2$. We now show that $J \leq(4 C A)^{5 / 2} M_{T}^{5(p-1) / 2}$ by the contradiction argument. Suppose $(4 C A)^{5 / 2} M_{T}^{5(p-1) / 2}<J \leq \infty$. We choose an integer $J^{\prime}$ so that $J^{\prime}=J$ if $J<\infty$ and $J^{\prime}=(4 C A)^{5 / 2} M_{T}^{5(p-1) / 2}+1$ if $J=\infty$.

For $0 \leq i \leq J^{\prime}$, define

$$
\begin{aligned}
f_{i}^{\omega}(x) & :=\left\|\left|\partial_{x}\right|^{s_{p}} u_{\omega}(\cdot, x)\right\|_{L_{t}^{10}\left[t_{i}, t_{i+1}\right]}, \quad f_{i}^{\infty}(x):=\left\|\left|\partial_{x}\right|^{s_{p}} U(\cdot, x)\right\|_{L_{t}^{10}\left[t_{i}, t_{i+1}\right]}, \\
g_{j}^{\omega}(x) & :=\left\|u_{\omega}(\cdot, x)\right\|_{L_{t}^{\frac{5}{2}(p-1)}\left[t_{i}, t_{i+1}\right]}, \quad g_{j}^{\infty}(x):=\|U(\cdot, x)\|_{L_{t}^{\frac{5}{2}(p-1)}\left[t_{i}, t_{i+1}\right]} .
\end{aligned}
$$


Since

$$
\begin{aligned}
\left\|u_{\omega}\right\|_{L_{x}^{\frac{5}{4}(p-1)} L_{T}^{\frac{5}{2}(p-1)}} & \geq\left(\left\|u_{\omega}(\cdot, x)\right\|_{L_{t}^{\frac{5}{2}(p-1)}\left(\left(0, t_{J^{\prime}}\right)\right)}^{\frac{5}{2}(p-1)}\right) \\
& =\|\left(\sum_{i=0}^{\frac{2}{5(p-1)}}\left|g_{i}^{\omega}(x)\right|_{\left.L_{x}^{\frac{5}{2}(p-1)}\right)^{\frac{5}{5(p-1)}}} \|_{L_{x}^{\frac{5}{4}(p-1)}}\right.
\end{aligned}
$$

we have

$$
\begin{aligned}
M_{T} \geq & \left\|\left(\sum_{i=0}^{J^{\prime}-1}\left|f_{i}^{\omega}(x)\right|^{10}\right)^{\frac{1}{10}}\right\|_{L_{x}^{5}}+\left\|\left(\sum_{i=0}^{J^{\prime}-1}\left|g_{i}^{\infty}(x)\right|^{10}\right)^{\frac{1}{10}}\right\|_{L_{x}^{5}} \\
& +\left\|\left(\sum_{i=0}^{J^{\prime}-1}\left|g_{i}^{\omega}(x)\right|^{\frac{5}{2}(p-1)}\right)^{\frac{2}{5(p-1)}}\right\|_{L_{x}^{\frac{5}{4}(p-1)}} \\
& +\left\|\left(\sum_{i=0}^{J^{\prime}-1}\left|g_{i}^{\infty}(x)\right|^{\frac{5}{2}(p-1)}\right)^{\frac{2}{5(p-1)}}\right\|_{L_{x}^{\frac{5}{4}(p-1)}} \cdot
\end{aligned}
$$

By the Hölder inequality, we obtain

$$
\begin{aligned}
\left(\frac{1}{4 C A}\right)^{\frac{1}{p-1}} J^{\prime} \leq & \sum_{i=0}^{J^{\prime}-1} M_{\left[t_{i}, t_{i+1}\right]} \leq\left(J^{\prime}\right)^{\frac{4}{5}}\left(\sum_{i=0}^{J^{\prime}-1}\left\|\left|\partial_{x}\right|^{s_{p}} u^{\omega}\right\|_{L_{x}^{5} L_{t}^{10}\left[t_{i}, t_{i+1}\right]}^{5}\right)^{\frac{1}{5}} \\
& +\left(J^{\prime}\right)^{\frac{4}{5}}\left(\sum_{i=0}^{J^{\prime}-1}\left\|\left|\partial_{x}\right|^{s_{p}} U^{\frac{1}{5}}\right\|_{L_{x}^{5} L_{t}^{10}\left[t_{i}, t_{i+1}\right]}^{5}\right. \\
& +\left(J^{\prime}\right)^{\frac{5 p-9}{5(p-1)}}\left(\sum_{i=0}^{J^{\prime}-1}\left\|u^{\omega}\right\|_{L_{x}^{\frac{5}{4}(p-1)} L_{t}^{\frac{5}{2}(p-1)}\left[t_{i}, t_{i+1}\right]}^{\frac{4}{5(p-1)}}\right)^{\frac{4}{5(p-1)}} \\
& +\left(J^{\prime}\right)^{\frac{5 p-9}{5(p-1)}}\left(\sum_{i=0}^{J^{\prime}-1}\|U\|^{\frac{5}{4}(p-1)}{ }_{L_{x}^{\frac{5}{4}(p-1)}}^{\frac{5}{2}(p-1)} L_{t}^{\frac{5}{2}\left(t_{i}, t_{i+1}\right]}\right.
\end{aligned}
$$

The right hand side of the above inequality can be rewritten as

$$
\begin{gathered}
\left(J^{\prime}\right)^{\frac{4}{5}}\left\|\left(\sum_{i=0}^{J^{\prime}-1}\left|f_{i}^{\omega}(x)\right|^{5}\right)^{\frac{1}{5}}\right\|\left\|_{L_{x}^{5}}+\left(J^{\prime}\right)^{\frac{4}{5}}\right\|\left(\sum_{i=0}^{J^{\prime}-1}\left|f_{i}^{\infty}(x)\right|^{5}\right)^{\frac{1}{5}} \|_{L_{x}^{5}} \\
+\left(J^{\prime}\right)^{\frac{5 p-9}{5(p-1)}}\left\|\left(\sum_{i=0}^{J^{\prime}-1}\left|g_{i}^{\omega}(x)\right|^{\frac{5}{4}(p-1)}\right)^{\frac{4}{5(p-1)}}\right\| \|_{L_{x}^{\frac{5}{4}(p-1)}} \\
+\left(J^{\prime}\right)^{\frac{5 p-9}{5(p-1)}}\left\|\left(\sum_{i=0}^{J^{\prime}-1}\left|g_{i}^{\infty}(x)\right|^{\frac{5}{4}(p-1)}\right)^{\frac{4}{5(p-1)}}\right\|_{L_{x}^{\frac{5}{4}(p-1)}} .
\end{gathered}
$$


The Hölder inequality and (5.1) yield

$$
\begin{aligned}
\left(\frac{1}{4 C A}\right)^{\frac{1}{p-1}} J^{\prime} \leq & \left(J^{\prime}\right)^{\frac{9}{10}}\left\|\left(\sum_{i=0}^{J^{\prime}-1}\left|f_{i}^{\omega}(x)\right|^{10}\right)^{\frac{1}{10}}\right\|_{L_{x}^{5}}+\left(J^{\prime}\right)^{\frac{9}{10}}\left\|\left(\sum_{i=0}^{J^{\prime}-1}\left|f_{i}^{\infty}(x)\right|^{10}\right)^{\frac{1}{10}}\right\|_{L_{x}^{5}} \\
& +\left(J^{\prime}\right)^{\frac{5 p-7}{5(p-1)}}\left\|\left(\sum_{i=0}^{J^{\prime}-1}\left|g_{i}^{\omega}(x)\right|^{\frac{5}{2}(p-1)}\right)^{\frac{2}{5(p-1)}}\right\| \|_{L_{x}^{\frac{5}{4}(p-1)}} \\
& +\left(J^{\prime}\right)^{\frac{5 p-7}{5(p-1)}}\left\|\left(\sum_{i=0}^{J^{\prime}-1}\left|g_{i}^{\infty}(x)\right|^{\frac{5}{2}(p-1)}\right)^{\frac{2}{5(p-1)}}\right\|_{L_{x}^{\frac{5}{x}(p-1)}} \\
\leq & \left(J^{\prime}\right)^{\frac{5 p-7}{5(p-1)}} M_{T} .
\end{aligned}
$$

Hence we obtain $J^{\prime} \leq(4 C A)^{5 / 2} M_{T}^{5(p-1) / 2}$. This contradicts the definition of $J^{\prime}$, which proves the claim.

\section{Acknowledgements}

We thank the referees for careful reading our manuscript and for giving useful comments.

\section{References}

[1] Abdullaev, F.K., Caputo J.G., Kraeukel, R.A., Malomed, B.A.: Controlling collapse in Bose-Einstein condensates by temporal modulation of the scattering length. Phys. Rev. A 67, 012605 (2003)

[2] Bourgain, J.: Fourier restriction phenomena for certain lattice subsets and applications to nonlinear evolution equations II. The KdV equation. Geom. Funct. Anal. 3, 209-262 (1993)

[3] Carvajal, X., Panthee, M., Scialom, M.: On the critical KDV equation with time-oscillating nonlinearity,. Differ. Integral Equ. 24, 541-567 (2011)

[4] Cazenave, T., Scialom, M.: A Schrödinger equation with time-oscillating nonlinearity. Rev. Mat. Complut. 23, 321-339 (2010)

[5] Christ, F.M., Weinstein, M.I.: Dispersion of small amplitude solutions of the generalized Korteweg-de Vries equation. J. Funct. Anal. 100, 87-109 (1991)

[6] Damergi, I., Goubet, O.: Blow-up solutions to the nonlinear Schrödinger equation with oscillating nonlinearities. J. Math. Anal. Appl. 352, 336-344 (2009)

[7] Dodson, B.: Global well-posedness and scattering for the defocusing, masscritical generalized KdV equation (2013). arXiv:1304.8025

[8] Fang, D., Han, Z.: A Schrödinger equation with time-oscillating critical nonlinearity. Nonlinear Anal. 74, 4698-4708 (2011)

[9] Farah, L.G., Pastor, A.: well-posedness and wave operator for the gKdV equation. Bull. Sci. Math. 137, 229-241 (2013) 
[10] Farah, L.G., Linares, F., Pastor, A.: The supercritical generalized KdV equation: global well-posedness in the energy space and below. Math. Res. Lett. 18, 357-377 (2011)

[11] Kato, T.: On the Cauchy problem for the (generalized) Korteweg-de Vries equation. Stud. Appl. Math. 8, 93-128 (1983)

[12] Kenig, C.E., Ponce, G., Vega, L.: Well-posedness and scattering results for the generalized Korteweg-de Vries equation via the contraction principle. Commun. Pure Appl. Math. 46, 527-620 (1993)

[13] Kenig, C.E., Ponce, G., Vega, L.: A bilinear estimate with applications to the KdV equation. J. Am. Math. Soc. 9, 573-603 (1996)

[14] Knickerbocker, C.J., Newell, A.C.: Internal solitary waves near a turning point . Phys. Lett. A 75, 326-330 (1979)

[15] Konotop, V.V., Pacciani, P.: Collapse of solutions of the nonlinear Schrödinger equation with a time dependent nonlinearity: application to the Base-Einstein condensates. Phys. Rev. Lett. 94, 240405 (2005)

[16] Korteweg, D.J., de Vries, G.: On the change of form of long waves advancing in a rectangular canal, and on a new type of long stationary waves. Philos. Mag. 39, 422-443 (1895)

[17] Killip, R., Kwon, S., Shao, S., Visan, M.: On the mass-critical generalized KdV equation. Discrete Contin. Dyn. Syst. 32, 191-221 (2012)

[18] Martel, Y., Merle, F.: Blow up in finite time and dynamics of blow up solutions for the critical generalized KdV equation. J. Am. Math. Soc. 15, 617-664 (2002)

[19] Masaki, S., Segata, J.: On well-posedness of generalized Korteweg-de Vries equation in scale critical $\hat{L}^{r}$ space. Anal. PDE 9, 699-725 (2016)

[20] Molinet, L., Ribaud, F.: On the Cauchy problem for the generalized Kortewegde Vries equation . Commun. Partial Differ. Equ. 28, 2065-2091 (2003)

[21] Molinet, L., Ribaud, F.: Well-posedness results for the generalized BenjaminOno equation with small initial data. J. Math. Pures Appl. 83, 277-311 (2004)

[22] Nunes, W.V.L.: Global well-posedness for the transitional Korteweg-de Vries equation. Appl. Math. Lett. 11, 15-20 (1998)

[23] Panthee, M., Scialom, M.: On the supercritical KdV equation with timeoscillating nonlinearity. Nonlinear Differ. Equ. Appl. 20, 1191-1212 (2013)

[24] Strunk, N.: Well-posedness for the supercritical gKdV equation. Commun. Pure Appl. Anal. 13, 527-542 (2014) 
Jun-ichi Segata and Keishu Watanabe Mathematical Institute

Tohoku University

6-3 Aoba

Aramaki, Aoba-ku

Sendai 980-8578

Japan

e-mail: segata@m.tohoku.ac.jp

Keishu Watanabe

e-mail: sb4m29@math.tohoku.ac.jp

Received: 20 April 2016.

Accepted: 9 August 2016. 